\title{
The effects of exposure to particulate matter and neighbourhood deprivation on gestational hypertension
}

\author{
Lisa C. Vinikoor-Imler ${ }^{a}$, Simone C. Gray ${ }^{b}$, Sharon E. Edwards ${ }^{b}$ and Marie Lynn Miranda ${ }^{b, c}$ \\ ${ }^{a}$ National Center for Environmental Assessment, US Environmental Protection Agency, Research Triangle Park, NC, ${ }^{b}$ Children's Environmental \\ Health Initiative, School of Natural Resources and Environment, and 'Department of Pediatrics, University of Michigan, Ann Arbor, MI, USA
}

Correspondence:

Dr Lisa C. Vinikoor-Imler, US Environmental Protection Agency, ATTN: MD B243-01, 109 T.W. Alexander Drive, Research Triangle Park, NC 27711, USA.

E-mail: vinikoor-imler.lisa@epa.gov

\section{Summary}

Vinikoor-Imler LC, Gray SC, Edwards SE, Miranda ML. The effects of exposure to particulate matter and neighbourhood deprivation on gestational hypertension. Paediatric and Perinatal Epidemiology 2012; 26: 91-100.

Gestational hypertension, pre-eclampsia and eclampsia are conditions that affect the health of both mothers and infants during and after pregnancy. Recent research indicates the importance of considering environmental, social and individual contributors to poor pregnancy outcomes. Our research examined particulate matter (PM) concentrations as one measure of environmental exposure and neighbourhood quality as one measure of the social environment. We used these measures, as well as maternal characteristics, to predict the risk of gestational hypertension (including pre-eclampsia and eclampsia). North Carolina Detailed Birth Record data for 2000-2003 were obtained and geocoded for all singleton births. Levels of $\mathrm{PM}_{10}$ and $\mathrm{PM}_{2.5}$ were determined using air quality data from the US Environmental Protection Agency. Information on a woman's residential neighbourhood was determined from 2000 Census data. Modified Poisson regression models clustered by tract were used to examine the associations between PM levels, neighbourhood deprivation and maternal characteristics with gestational hypertension. Analysis was restricted to women residing within $20 \mathrm{~km}$ of a PM monitor. Both $\mathrm{PM}_{10}$ and $\mathrm{PM}_{2.5}$ were associated with gestational hypertension; the risk ratios for an interquartile range (IQR) increase in exposure were 1.07 [95\% confidence interval (CI) 1.04, 1.11] for $\mathrm{PM}_{10}$ (IQR: $3.92 \mu \mathrm{g} / \mathrm{m}^{3}$ ) and 1.11 [95\% CI 1.08, 1.15] for PM 2.5 (IQR: $\left.2.24 \mu \mathrm{g} / \mathrm{m}^{3}\right)$. Living in a neighbourhood with increased levels of deprivation was also associated with gestational hypertension. Any smoking during pregnancy, younger age and higher level of education were inversely associated with risk of gestational hypertension. Compared with non-Hispanic White women, non-Hispanic Black women were at higher risk of gestational hypertension, whereas Hispanic women were at lower risk. Increased levels of PM and neighbourhood deprivation, as well as certain individual characteristics, were associated with higher risk of gestational hypertension.

Keywords: air pollution, social deprivation, gestational hypertension, prenatal environment, particulates.

\section{Introduction}

Hypertensive disorders during pregnancy [gestational hypertension, pre-eclampsia and eclampsia, hereafter referred to as gestational hypertension (GH)] are detrimental to short-term and long-term health of both the mother and child. Pre-eclampsia/eclampsia is one of the leading causes of maternal and perinatal death. ${ }^{1-3}$
Infants born to mothers with $\mathrm{GH}$ are more likely to be small for gestational age, preterm and have low birthweight. ${ }^{4,5}$ They have also been shown to have higher rates of admission to neonatal intensive care units $^{6}$ and increased risk of hospitalisation for infectious, nervous, respiratory, endocrine, metabolic and blood/blood-forming organ diseases. ${ }^{7}$ Children of mothers with GH are more likely to have elevated 
blood pressure during adolescence, ${ }^{8,9}$ and an increased risk of stroke during adulthood. ${ }^{5}$ Having GH during a pregnancy has also been associated with increased risk of cardiovascular disease, stroke and Type II diabetes later in life. ${ }^{1,10-12}$

In the US, the rates of GH have been rising in recent years. ${ }^{13}$ In 2003-2004, the incidence rates were $30.6 / 1000$ for gestational hypertension (without preeclampsia) and 29.4/1000 for pre-eclampsia. ${ }^{13}$ Prior studies have shown that previous pregnancy ${ }^{14-16}$ and maternal race/ethnicity ${ }^{17}$ affect the rates of GH. In addition, a few studies have examined socio-economic status of the maternal residential neighbourhood, but the results have been inconsistent. ${ }^{18-20}$

Auchincloss et al. examined the association between mean levels of particulate matter (PM) with mean aerodynamic diameters $\leq 2.5 \mu \mathrm{m}\left(\mathrm{PM}_{2.5}\right)$ over 30 - and 60-day intervals and general blood pressure levels. ${ }^{21}$ The study demonstrated a change in hypertension measures with increases in $\mathrm{PM}_{2.5}$. It is plausible that if long-term $\mathrm{PM}_{2.5}$ exposure levels increase the risk of higher blood pressure levels, women exposed during their pregnancy to high $\mathrm{PM}_{2.5}$ exposure levels may be at increased risk of GH. The relationship between GH and air pollution, specifically PM, has been explored in a limited number of studies. A recent study reported a positive association between $\mathrm{PM}_{2.5}$ and preeclampsia. ${ }^{22}$ A prospective cohort study in the Netherlands also reported an increased risk of $\mathrm{GH}$ with higher $\mathrm{PM}_{10}$ concentrations. ${ }^{23}$

A recent article by Miranda et al. described pregnancy outcomes as the result of the forces of three sides of an integrated triangle, with environmental factors, social factors and host factors each forming one side of the triangle..$^{24}$ Our paper utilises that same conceptual framework. We examine the associations between an environmental factor (PM), a social factor (neighbourhood deprivation) and host factors (such as age and parity) with GH. We used data on women living in North Carolina and giving birth to a singleton infant between 2000 and 2003. The results of this study can be used to elucidate the social and environmental factors that may contribute to $\mathrm{GH}$.

\section{Methods}

\section{Birth data}

North Carolina Detailed Birth Record data were obtained from the North Carolina State Center for
Health Statistics. The data included all registered births occurring in North Carolina between 1 January 2000 and 31 December $2003(n=467780)$. Data from birth records were also used to determine whether a woman had GH, pre-eclampsia and/or eclampsia during her pregnancy. These were combined to form one variable representing $\mathrm{GH}$, similar to previous research. ${ }^{17}$ Information on maternal age, education, race/ethnicity, marital status, parity and smoking during pregnancy was also obtained from birth records.

In addition, the mother's residential address at birth was geocoded at the street level using ArcGIS 9.3 software (ESRI, Redlands, CA, USA) with a geocoding success rate of about $91 \%$ for women living in a county with a PM monitor ( $86 \%$ overall success rate). Women whose addresses could not be geocoded were excluded from further analysis $(n=67367)$. All aspects of the study were conducted in accordance with a human subjects research protocol approved by the Institutional Review Board.

\section{Air data}

Data on $\mathrm{PM}_{10}$ and $\mathrm{PM}_{2.5}$ levels were downloaded from the US Environmental Protection Agency's Air Quality System (AQS) for 1999-2003. ${ }^{25}$ Daily 24-h average concentrations were obtained every 1, 3 or 6 days from active monitors across North Carolina. During the study period there were 27-37 active $\mathrm{PM}_{10}$ monitors and 37-41 active $\mathrm{PM}_{2.5}$ monitors (Figure 1).

Residential addresses were linked to the closest active monitors. Birth date and gestational age were used to determine the start and end dates of the full gestational period for each infant, allowing PM exposure to be calculated as the 24-h values averaged over the full gestational period. In order to ensure that PM exposure could be sufficiently characterised, births were excluded if data were available for $<75 \%$ of the weeks of the entire gestational period. Births were also excluded if PM data were not available for two or more consecutive weeks of gestation. For births with data available for at least $75 \%$ of the gestational period and no missing data in consecutive weeks, any weeks of gestation with missing PM data were imputed using the average of the PM monitoring value of the previous and following weeks. Additionally, women were excluded from the study if they lived more than $20 \mathrm{~km}$ from an AQS monitor. Overall, the criteria based on sufficient monitoring data and proximity to 


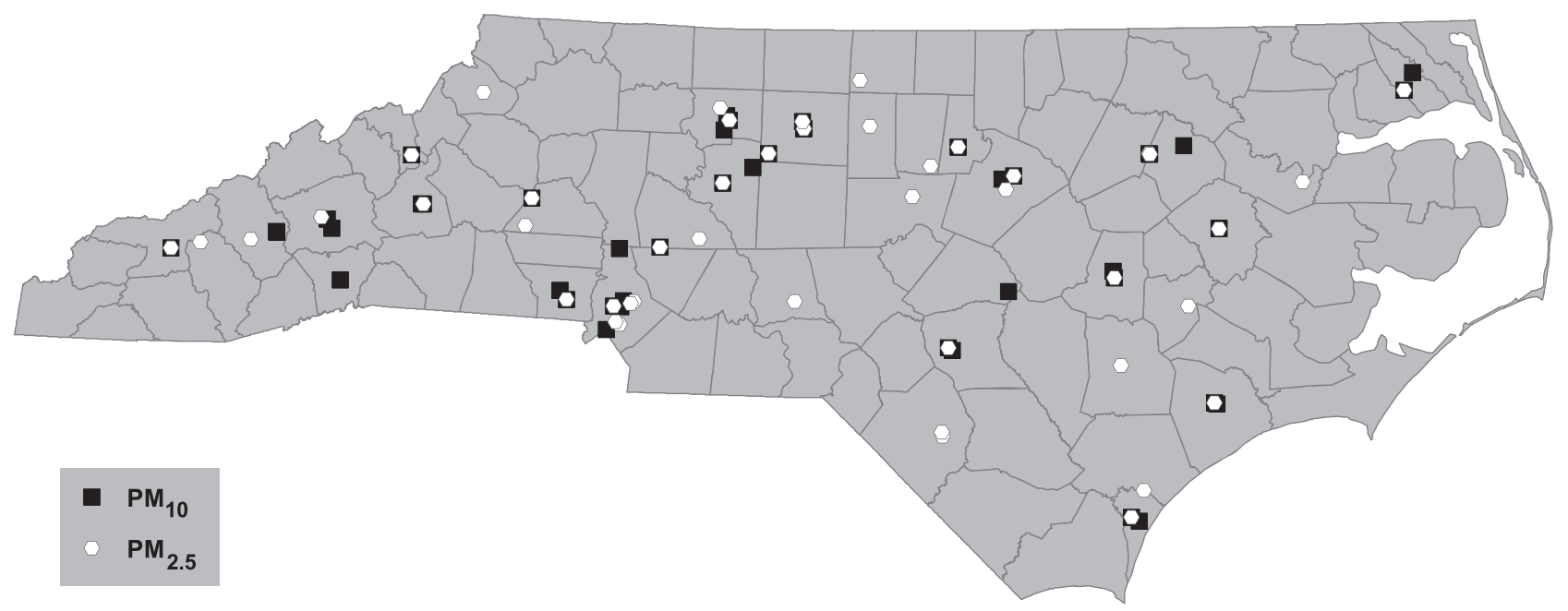

Figure 1. Locations of active $\mathrm{PM}_{10}$ and $\mathrm{PM}_{2.5}$ monitors during the study period.

the monitor resulted in the exclusion of 146907 women (approximately $37 \%$ of geocoded women).

\section{Neighbourhood data}

Tract-level data from the 2000 Census were utilised to create the Neighbourhood Deprivation Index (NDI). ${ }^{26}$ This index has been used previously as a measure of neighbourhood poverty in studies of birth outcomes. ${ }^{26-29}$ The NDI was created using the first factor loadings from a principal components analysis of the following census variables: per cent of households in poverty, per cent of female-headed households with dependents, per cent with household income $<\$ 30000$, per cent of households on public assistance, per cent of males in management/professional occupation, per cent living in crowded housing, per cent unemployed and per cent without a high school education. Quartiles were created for the NDI based on the distribution among census tracts captured by the 20-km buffers around $\mathrm{PM}_{10}$ and $\mathrm{PM}_{2.5}$ monitors. Women were assigned the NDI quartile for their census tract. Quartiles were created at the census tract level, as opposed to quartiles based on the population distribution, to avoid weighting the quartiles of NDI by population of the census tracts.

\section{Maternal characteristics}

Information on maternal characteristics was obtained from the birth records. The variable representing parity was dichotomised based on whether the infant was a first or higher-order birth. Smoking during pregnancy and marital status were also dichotomous variables. Maternal race was categorised as non-Hispanic White (NHW), non-Hispanic Black (NHB) and Hispanic (because of small numbers, women of other races/ ethnicities were excluded from the study, $n=10437$ ). The following categories were used for maternal education: <9th grade, attended some high school, completed high school, attended some college and completed college or a higher degree. Maternal age at delivery was categorised in 5-year increments for women between 15 and 44, with two additional categories for $<15$ years old and $>44$ years old.

Multi-fetal births $(n=8441)$ and infants with congenital abnormalities $(n=1711)$ were excluded from the analysis. Women who had more than three previous births were also excluded $(n=6571)$. Infants with a birthweight $<400 \mathrm{~g}(n=235)$ or a gestational age $<24$ or $>42$ weeks $(n=461)$ were removed from the study. Additionally, women were excluded if data on key variables were missing $(n=622)$ or they reported having chronic hypertension $(n=2253)$. Figure 2 depicts the process of excluding individuals from the study population. Under these restrictions, a total of 222775 births were available for analysis.

\section{Statistical analysis}

We used modified Poisson regression models with robust standard errors clustered by census tract to assess the associations between $\mathrm{GH}$ and $\mathrm{PM}_{10}$, $\mathrm{PM}_{2.5}$, maternal characteristics and neighbourhood 


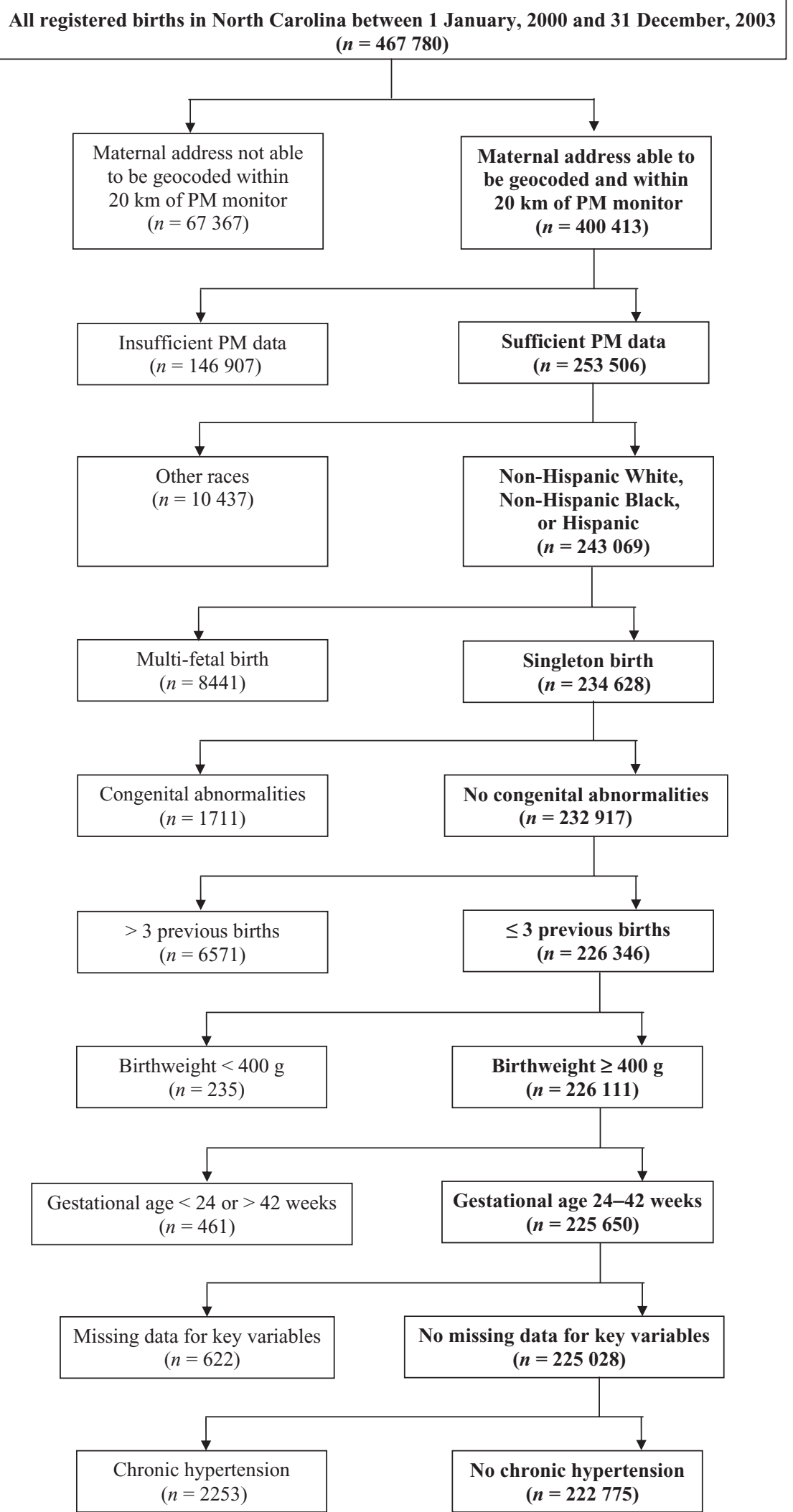

Figure 2. Flowchart of inclusion criteria for the study population. PM, particulate matter. 
deprivation. $\mathrm{PM}_{10}$ and $\mathrm{PM}_{2.5}$ were run as continuous variables in separate models with the same covariates (NDI quartiles and maternal characteristics as described above) included in both models. A linear specification was used for $\mathrm{PM}_{10}$ and $\mathrm{PM}_{2.5}$ after it was determined that alternate specifications, such as quadratic, did not improve the fit of the model. Clustering by census tract was performed to account for the inclusion of a tract-conditional variable, NDI, in the models. All models were run using SAS 9.2 (SAS Institute Inc., Cary, NC, USA). Additionally, models including interactions of $\mathrm{PM}_{10}$ and $\mathrm{PM}_{2.5}$ with level of neighbourhood deprivation were included to observe how the associations varied by level of deprivation. This was done by creating an interaction variable that combined PM concentration with a dichotomous variable for neighbourhood deprivation. The interaction was assessed using a likelihood ratio test and results for each deprivation category are presented.

The models were restricted to women living within $20 \mathrm{~km}$ of a monitor. To check the sensitivity of our findings to the distance of maternal residence to the PM monitors, models were also run restricting to women living within $10 \mathrm{~km}$ of a monitor and women living within $5 \mathrm{~km}$ of a monitor. The results did not substantially differ across the buffer sizes. Therefore, we only present results for the women living within $20 \mathrm{~km}$ of a monitor.

\section{Results}

The total study population after the exclusion criteria were applied was 222775 women. Of the women in the study population, $5.4 \%$ had GH ( $n=12085)$. Women with $\mathrm{GH}$ had similar mean concentrations of $\mathrm{PM}_{10}$ and $\mathrm{PM}_{2.5}$ exposure during pregnancy compared with normotensive women. The mean $\mathrm{PM}_{10}$ levels during pregnancy were $22.4 \mu \mathrm{g} / \mathrm{m}^{3}$ among women with $\mathrm{GH}$ and $22.2 \mu \mathrm{g} / \mathrm{m}^{3}$ among women without $\mathrm{GH}$. The respective mean $\mathrm{PM}_{2.5}$ levels were $14.7 \mu \mathrm{g} / \mathrm{m}^{3}$ and $14.5 \mu \mathrm{g} / \mathrm{m}^{3}$. A higher proportion of normotensive women were Hispanic (13.4\% vs. 9.2\%) and a higher proportion had previously given birth (56.9\% vs. $38.3 \%$ ). Maternal age and marital status were similar between the two groups (Table 1).

Results of the regression analysis are shown in Table 2. Positive associations were detected between $\mathrm{PM}_{2.5}$ and $\mathrm{GH}$; the risk ratio (RR) for an increase in $\mathrm{PM}_{2.5}$ equivalent to the sample's interquartile range (IQR) $\left(2.24 \mu \mathrm{g} / \mathrm{m}^{3}\right)$ was 1.11 [95\% confidence interval
(CI) 1.08, 1.15]. Similarly, there was an association between $\mathrm{PM}_{10}$ and GH [RR 1.07 [95\% CI 1.04, 1.11] per increase in $\mathrm{PM}_{10}$ equivalent to the IQR $\left.\left(3.92 \mu \mathrm{g} / \mathrm{m}^{3}\right)\right]$.

Compared with women aged 25-29, women aged 35 years and older were more likely to present with $\mathrm{GH}$, and women under 25 years old were less likely to present with GH. Although no association was seen for most levels of education, women who completed at least a college degree had a lower risk of having $\mathrm{GH}$ compared with women completing a high school degree. GH was also inversely associated with maternal smoking, and the risk of GH was elevated among primiparous women. Compared with NHW women, NHB women had a higher risk of GH, whereas Hispanic women had a decreased risk. Marital status showed no association with GH.

The NDI was calculated using principal components analysis and was found to explain $60 \%$ of the variance of the census tract variables (alpha $=0.90)$. Increased risk of GH was associated with higher NDI values; that is, increased neighbourhood deprivation above the first quartile was positively associated with $\mathrm{GH}$ (Table 2). We further examined the role of NDI by assessing how deprivation affects the association between $\mathrm{GH}$ and concentrations of $\mathrm{PM}_{10}$ and $\mathrm{PM}_{2.5}$. Because of the similarity of the associations between the second, third and fourth quartiles for NDI, these were collapsed into one category. No interaction was present between NDI categories and $\mathrm{PM}_{2.5}(P$-value for likelihood ratio test 0.24 ). Using interaction models, the adjusted RRs for each IQR increase in $\mathrm{PM}_{2.5}$ were 1.08 [95\% CI 1.02, 1.14] for women residing in areas with the lowest deprivation and 1.12 [95\% CI 1.08, 1.17] for those in the combined upper $75 \%$ of the NDI. A more pronounced difference across NDI levels was observed for $\mathrm{PM}_{10}$ and $\mathrm{GH}$ (lowest neighbourhood deprivation: RR 1.02 [95\% CI 0.98, 1.07] per IQR increase; upper $75 \%$ of neighbourhood deprivation: RR 1.10 [95\% CI 1.06, 1.14] per IQR increase; $P$-value for likelihood ratio test $<0.05)$. Despite the changes in the association, PM levels were similar among all categories of the NDI with $\mathrm{PM}_{10}$ concentrations in the lowest quartile of deprivation being only slightly higher than the other quartiles (Table 3).

\section{Discussion}

Following the conceptual framework laid out in previous work on the multiple factors affecting pregnancy outcomes, ${ }^{24}$ this study examined the association of $\mathrm{GH}$ 
Table 1. Demographic characteristics of women with exposure information concerning $\mathrm{PM}_{10}$ and/or $\mathrm{PM}_{2.5}$ by gestational hypertension status

\begin{tabular}{|c|c|c|c|}
\hline & $\begin{array}{l}\text { Gestational } \\
\text { hypertension } \\
(n=12085)\end{array}$ & $\begin{array}{l}\text { No gestational } \\
\text { hypertension } \\
(n=210690)\end{array}$ & $\begin{array}{c}\text { Total } \\
(n=222775)\end{array}$ \\
\hline \multicolumn{4}{|c|}{$\mathrm{PM}_{10}$ during pregnancy $\left(\mu \mathrm{g} / \mathrm{m}^{3}\right)$} \\
\hline Mean (SD) & $22.4(3.6)$ & $22.2(3.6)$ & $22.2(3.6)$ \\
\hline Median & 22.0 & 21.9 & 21.9 \\
\hline Interquartile range & $20.1,24.1$ & $20.0,23.9$ & $20.0,23.9$ \\
\hline \multicolumn{4}{|c|}{$\mathrm{PM}_{2.5}$ during pregnancy $\left(\mu \mathrm{g} / \mathrm{m}^{3}\right)$} \\
\hline Mean (SD) & $14.7(1.7)$ & $14.5(1.7)$ & $14.5(1.7)$ \\
\hline Median & 14.7 & 14.5 & 14.5 \\
\hline \multirow[t]{2}{*}{ Interquartile range } & $13.6,15.8$ & $13.5,15.7$ & $13.5,15.7$ \\
\hline & $n(\%)$ & $n(\%)$ & $n(\%)$ \\
\hline \multicolumn{4}{|l|}{ Neighbourhood } \\
\hline \multicolumn{4}{|l|}{ Deprivation Index } \\
\hline 1st quartile & $3290(27.2)$ & $65769(31.2)$ & $69059(31.0)$ \\
\hline 2nd quartile & $3352(27.7)$ & $54548(25.9)$ & $57900(26.0)$ \\
\hline 3rd quartile & $2943(24.4)$ & $47024(22.3)$ & $49967(22.4)$ \\
\hline 4th quartile & $2500(20.7)$ & $43349(20.6)$ & 45849 (20.6) \\
\hline \multicolumn{4}{|l|}{ Race } \\
\hline Non-Hispanic White & $7468(61.8)$ & $129670(61.5)$ & $137138(61.6)$ \\
\hline Non-Hispanic Black & $3506(29.0)$ & $52769(25.0)$ & $56275(25.3)$ \\
\hline Hispanic & $1111(9.2)$ & $28251(13.4)$ & $29362(13.2)$ \\
\hline \multicolumn{4}{|l|}{ Previous birth } \\
\hline No & $7457(61.7)$ & $90810(43.1)$ & $98267(44.1)$ \\
\hline Yes & $4628(38.3)$ & $119880(56.9)$ & $124508(55.9)$ \\
\hline \multicolumn{4}{|c|}{ Smoked during pregnancy } \\
\hline No & $10969(90.8)$ & $187411(89.0)$ & $198380(89.0)$ \\
\hline Yes & $1116(9.2)$ & $23279(11.0)$ & $24395(11.0)$ \\
\hline \multicolumn{4}{|l|}{ Maternal age (years) } \\
\hline$<15$ & $40(0.3)$ & $464(0.2)$ & $504(0.2)$ \\
\hline $15-19$ & $1474(12.2)$ & $22658(10.8)$ & $24132(10.8)$ \\
\hline $20-24$ & $2987(24.7)$ & $55517(26.4)$ & $58504(26.3)$ \\
\hline $25-29$ & $3293(27.3)$ & $56189(26.7)$ & $59482(26.7)$ \\
\hline $30-34$ & $2730(22.6)$ & $51163(24.3)$ & $53893(24.2)$ \\
\hline $35-39$ & $1286(10.6)$ & $21109(10.0)$ & $22395(10.1)$ \\
\hline $40-44$ & $264(2.2)$ & $3480(1.7)$ & $3744(1.7)$ \\
\hline$\geq 45$ & $11(0.1)$ & $108(0.1)$ & $119(0.1)$ \\
\hline \multicolumn{4}{|l|}{$\begin{array}{l}\text { Maternal education } \\
\text { completed }\end{array}$} \\
\hline$<9$ th grade & $502(4.2)$ & $12060(5.7)$ & $12562(5.6)$ \\
\hline Some high school & 1579 (13.1) & $29221(13.9)$ & $30800(13.8)$ \\
\hline High school degree & $3490(28.9)$ & $57301(27.2)$ & $60791(27.3)$ \\
\hline Some college & $3052(25.3)$ & 45978 (21.8) & $49030(22.0)$ \\
\hline College degree & $3462(28.6)$ & $66130(31.4)$ & 69592 (31.2) \\
\hline \multicolumn{4}{|l|}{ Marital status } \\
\hline Married & $8020(66.4)$ & $142634(67.7)$ & 150654 (67.6) \\
\hline Not married & 4065 (33.6) & $68056(32.3)$ & 72121 (32.4) \\
\hline
\end{tabular}

with PM exposure during pregnancy, neighbourhood deprivation and maternal host factors.

We found positive associations between $\mathrm{PM}_{2.5}$ and $\mathrm{PM}_{10}$ concentrations and the risk of $\mathrm{GH}$. This is especially noteworthy given the relatively low $\mathrm{PM}_{2.5}$ and
$\mathrm{PM}_{10}$ concentrations found in North Carolina compared with other regions of the US. Our findings are similar to a study by $\mathrm{Wu}$ et al., which reported a positive association between $\mathrm{PM}_{2.5}$ and pre-eclampsia among women living in southern California. ${ }^{22}$ 


\begin{tabular}{|c|c|c|}
\hline & \multicolumn{2}{|c|}{ Risk ratio $[95 \% \mathrm{CI}]$} \\
\hline & $\begin{array}{l}\text { Model including } \mathrm{PM}_{2.5} \\
\quad(n=214325)\end{array}$ & $\begin{array}{l}\text { Model including } \mathrm{PM}_{10} \\
\quad(n=159358)\end{array}$ \\
\hline PM per interquartile range increase ${ }^{\mathrm{b}}$ & $1.11[1.08,1.15]$ & $1.07[1.04,1.11]$ \\
\hline \multicolumn{3}{|l|}{ Neighbourhood Deprivation Index } \\
\hline 1st quartile & 1.00 Reference & 1.00 Reference \\
\hline 2nd quartile & $1.23[1.15,1.32]$ & $1.25[1.16,1.36]$ \\
\hline 3rd quartile & $1.30[1.20,1.40]$ & $1.34[1.23,1.46]$ \\
\hline 4th quartile & $1.24[1.14,1.35]$ & $1.27[1.16,1.39]$ \\
\hline \multicolumn{3}{|l|}{ Maternal age (years) } \\
\hline$<15$ & $0.79[0.57,1.10]$ & $0.82[0.56,1.20]$ \\
\hline $15-19$ & $0.72[0.67,0.78]$ & $0.70[0.64,0.77]$ \\
\hline $20-24$ & $0.78[0.74,0.83]$ & $0.77[0.72,0.82]$ \\
\hline $25-29$ & 1.00 Reference & 1.00 Reference \\
\hline $30-34$ & $1.05[1.00,1.11]$ & $1.06[1.00,1.13]$ \\
\hline $35-39$ & $1.29[1.21,1.37]$ & $1.29[1.20,1.39]$ \\
\hline $40-44$ & $1.56[1.37,1.76]$ & $1.59[1.37,1.85]$ \\
\hline$\geq 45$ & $1.95[1.08,3.52]$ & $1.69[0.67,3.68]$ \\
\hline \multicolumn{3}{|l|}{ Maternal education } \\
\hline$<9$ th grade & $0.96[0.87,1.07]$ & $0.96[0.86,1.09]$ \\
\hline Completed some high school & $0.99[0.93,1.06]$ & $1.01[0.93,1.09]$ \\
\hline Completed high school & 1.00 Reference & 1.00 Reference \\
\hline Completed some college & $0.98[0.93,1.03]$ & $0.97[0.91,1.03]$ \\
\hline Completed college & $0.71[0.67,0.76]$ & $0.70[0.65,0.75]$ \\
\hline \multicolumn{3}{|l|}{ Smoked during pregnancy } \\
\hline No & 1.00 Reference & 1.00 Reference \\
\hline Yes & $0.80[0.74,0.85]$ & $0.84[0.78,0.91]$ \\
\hline \multicolumn{3}{|l|}{ Previous birth } \\
\hline Yes & 1.00 Reference & 1.00 Reference \\
\hline No & $2.28[2.19,2.37]$ & $2.28[2.18,2.39]$ \\
\hline \multicolumn{3}{|l|}{ Race } \\
\hline Non-Hispanic White & 1.00 Reference & 1.00 Reference \\
\hline Non-Hispanic Black & $1.15[1.10,1.21]$ & $1.14[1.08,1.21]$ \\
\hline Hispanic & $0.67[0.61,0.73]$ & $0.67[0.61,0.73]$ \\
\hline \multicolumn{3}{|l|}{ Marital status } \\
\hline Married & 1.00 Reference & 1.00 Reference \\
\hline Not married & $0.97[0.92,1.03]$ & $0.98[0.92,1.05]$ \\
\hline
\end{tabular}

${ }^{\mathrm{a}}$ Models included all variables listed above.

${ }^{b}$ Interquartile range is $3.92 \mu \mathrm{g} / \mathrm{m}^{3}$ for $\mathrm{PM}_{10}$ and $2.24 \mu \mathrm{g} / \mathrm{m}^{3}$ for $\mathrm{PM}_{2.5}$.

\begin{tabular}{lcccc}
\hline & \multicolumn{4}{c}{ Neighbourhood Deprivation Index } \\
\cline { 2 - 5 } & Quartile 1 & Quartile 2 & Quartile 3 & Quartile 4 \\
\hline $\mathrm{PM}_{10}$ during pregnancy $\left(\mu \mathrm{g} / \mathrm{m}^{3}\right)$ & & & & \\
$n$ & 53726 & 40191 & 33154 & 32287 \\
Mean (SD) & $23.00(3.74)$ & $21.63(3.27)$ & $21.75(3.73)$ & $21.88(3.59)$ \\
Median & 22.39 & 21.50 & 21.58 & 21.67 \\
Interquartile range & $20.80,24.65$ & $19.69,23.26$ & $19.48,23.74$ & $19.66,23.79$ \\
PM $_{2.5}$ during pregnancy $\left(\mu \mathrm{g} / \mathrm{m}^{3}\right)$ & & & & \\
$n$ & 65912 & 55454 & 48356 & 44605 \\
Mean (SD) & $14.64(1.59)$ & $14.47(1.73)$ & $14.40(1.83)$ & $14.44(1.83)$ \\
Median & 14.59 & 14.49 & 14.46 & 14.49 \\
Interquartile range & $13.70,15.62$ & $13.41,15.65$ & $13.23,15.67$ & $13.19,15.74$ \\
\hline
\end{tabular}

Table 2. Risk ratios [95\% confidence intervals] for the associations between particulate matter (PM) exposure during pregnancy, maternal and neighbourhood characteristics and gestational hypertension $^{\mathrm{a}}$
Table 3. Distribution of $\mathrm{PM}_{10}$ and $\mathrm{PM}_{2.5}$ by quartile of Neighbourhood Deprivation Index

Published 2011. This article is a U.S. Government work and is in the public domain in the USA.

Paediatric and Perinatal Epidemiology, 2012, 26, 91-100 
Additionally, among women in the Netherlands, higher $\mathrm{PM}_{10}$ concentrations were associated with increased risk of GH and increased systolic blood pressure measurements in the second and third trimesters. ${ }^{23}$ Further work is needed to determine if the association persists in other study populations and to describe the potential mechanism(s) associating PM with GH.

We also observed a positive relationship between neighbourhood deprivation and GH. A few studies have examined socio-economic status of the maternal residential neighbourhood, but the results have been inconsistent. Agyemang et al. reported no association between neighbourhood median income or unemployment levels and GH. ${ }^{18}$ Another study examining the difference between two areas of Oslo, Norway reported higher rates of pre-eclampsia in areas with lower incomes. ${ }^{19}$ Conversely, a study conducted in Sweden reported higher rates of pre-eclampsia in high-income areas. ${ }^{20}$ Studies on chronic hypertension have reported positive associations with neighbourhood deprivation. ${ }^{18,30}$ In addition, in our study the association between $\mathrm{PM}_{10}$ levels and $\mathrm{GH}$ varied within categories of neighbourhood deprivation. The association between $\mathrm{PM}_{10}$ concentration and $\mathrm{GH}$ was greater among those living in neighbourhoods with the highest deprivation compared with those neighbourhoods that had lower levels of neighbourhood deprivation. However, no difference was observed between the two deprivation categories for the association between $\mathrm{PM}_{2.5}$ concentration and GH. Future studies of PM and neighbourhood deprivation in relation to GH may help elucidate whether social factors, such as neighbourhood deprivation, influence the association between PM and GH.

Associations were detected with other covariates included in the models. Older maternal age, having no previous births and neighbourhood deprivation were positively associated with GH. Smoking during pregnancy, younger age and high levels of education were inversely associated with GH. Compared with NHW women, the risk of having GH was lower among Hispanic women, but higher among NHB women. Some of these associations have been previously demonstrated. Prior work examining maternal education found that GH was inversely associated with education leve ${ }^{31}$ and that $\mathrm{GH}$ varied by race, with African Americans having higher odds and Hispanics having lower odds. ${ }^{17,32}$ Another study of women giving birth in North Carolina reported a positive association between maternal age and hypertension, although this association had a strong interaction with race/ ethnicity. ${ }^{17}$ Wallis et al. also reported a slight positive association between $\mathrm{GH}$ and older maternal age. ${ }^{13}$ However, these researchers also reported higher risk of GH at younger ages, which was not observed in our study where younger age appeared to reduce the odds of GH. The inverse relationship reported in this study between smoking during pregnancy and $\mathrm{GH}$ has been reported in multiple studies, although the mechanism for this association is unknown. ${ }^{33-35}$ This study also detected higher risk of $\mathrm{GH}$ among primiparous women. Prior research supports the associations between GH and previous pregnancies. ${ }^{14-16}$

This study is not without limitations. PM exposure was averaged over the whole pregnancy regardless of when the GH began. Future studies that match the time of diagnosis for GH during pregnancy with temporal data on air pollution may elucidate this association further. In addition, women were assigned PM exposure levels based on their location of residence; however, no direct sampling was performed to gauge their actual exposure. The actual exposure could vary based on time spent at different locations and doing various activities (such as time spent in traffic during a commute). No wind or other meteorological variables were considered in the determination of PM exposure concentrations. By assigning all women within a given 20-km buffer the same PM monitor value, we may not be accounting for locally varying increased exposures or hot-spots within the radial buffer. However, a sensitivity analysis detected similar results at smaller distances from the monitors ( 5 and $10 \mathrm{~km}$ ). This similarity of results across a range of buffers was also observed in another study of this population. ${ }^{36}$ Additionally, information was not available on residential mobility during pregnancy, only residence at delivery, so it is possible that some women included in the study lived elsewhere and were exposed to different PM levels during part of their pregnancy. Finally, information on body mass index, which could have been associated with both neighbourhood deprivation and the outcome, was not available.

Despite these limitations, there are multiple strengths of the study. First, it includes a large number of women from across North Carolina. Additionally, multiple risk factors were simultaneously considered; air pollution and neighbourhood deprivation were examined while controlling for multiple covariates that potentially confound the associations. Finally, information on $\mathrm{GH}$, as well as other maternal characteristics, 
was provided from vital statistics records, which has been shown to have good reliability. ${ }^{37}$

In summary, a conceptual framework proposed that environmental, social and host factors all contribute to reproductive outcomes, ${ }^{24}$ which was true in this study. Among women giving birth in North Carolina between 2000 and 2003, higher levels of both $\mathrm{PM}_{10}$ and $\mathrm{PM}_{2.5}$ and higher levels of neighbourhood deprivation were positively associated with GH. Additional maternal characteristics also affected the risk of a woman having GH. Future research on the mechanisms by which air pollution and neighbourhood deprivation, or other measures of social and environmental stress, are associated with GH has the potential to improve shortand long-term health of women and their children.

\section{Acknowledgements}

Dr Miranda, Dr Gray and Ms Edwards were supported in part by a grant from the US Environmental Protection Agency (RD-83329301).

\section{Disclaimer}

The views expressed in this article are those of the authors and do not necessarily reflect the views or policies of the US Environmental Protection Agency.

\section{References}

1 Duley L. The global impact of pre-eclampsia and eclampsia. Seminars in Perinatology 2009; 33:130-137.

2 Koonin LM, MacKay AP, Berg CJ, Atrash HK, Smith JC. Pregnancy-related mortality surveillance - United States, 1987-1990. MMWR. CDC Surveillance Summaries 1997; 46:17-36.

3 Ngoc NT, Merialdi M, Abdel-Aleem H, Carroli G, Purwar $\mathrm{M}$, Zavaleta $\mathrm{N}$, et al. Causes of stillbirths and early neonatal deaths: data from 7993 pregnancies in six developing countries. Bulletin of the World Health Organization 2006; 84:699-705.

4 Allen VM, Joseph K, Murphy KE, Magee LA, Ohlsson A. The effect of hypertensive disorders in pregnancy on small for gestational age and stillbirth: a population based study. BMC Pregnancy and Childbirth 2004; 4:17.

5 Kajantie E, Eriksson JG, Osmond C, Thornburg K, Barker DJ. Pre-eclampsia is associated with increased risk of stroke in the adult offspring: the Helsinki birth cohort study. Stroke 2009; 40:1176-1180.

6 Hauth JC, Ewell MG, Levine RJ, Esterlitz JR, Sibai B, Curet LB, et al. Pregnancy outcomes in healthy nulliparas who developed hypertension. Calcium for Preeclampsia
Prevention Study Group. Obstetrics and Gynecology 2000; 95:24-28.

7 Wu CS, Nohr EA, Bech BH, Vestergaard M, Catov JM, Olsen J. Health of children born to mothers who had preeclampsia: a population-based cohort study. American Journal of Obstetrics and Gynecology 2009; 201:269, e1-e10.

8 Tenhola S, Rahiala E, Halonen P, Vanninen E, Voutilainen R. Maternal preeclampsia predicts elevated blood pressure in 12-year-old children: evaluation by ambulatory blood pressure monitoring. Pediatric Research 2006; 59:320-324.

9 Vatten LJ, Romundstad PR, Holmen TL, Hsieh CC, Trichopoulos D, Stuver SO. Intrauterine exposure to preeclampsia and adolescent blood pressure, body size, and age at menarche in female offspring. Obstetrics and Gynecology 2003; 101:529-533.

10 Bauer ST, Cleary KL. Cardiopulmonary complications of pre-eclampsia. Seminars in Perinatology 2009; 33:158-165.

11 Bellamy L, Casas JP, Hingorani AD, Williams DJ. Pre-eclampsia and risk of cardiovascular disease and cancer in later life: systematic review and meta-analysis. BMJ 2007; 335:974.

12 Lykke JA, Langhoff-Roos J, Sibai BM, Funai EF, Triche EW, Paidas MJ. Hypertensive pregnancy disorders and subsequent cardiovascular morbidity and type 2 diabetes mellitus in the mother. Hypertension 2009; 53:944-951.

13 Wallis AB, Saftlas AF, Hsia J, Atrash HK. Secular trends in the rates of preeclampsia, eclampsia, and gestational hypertension, United States, 1987-2004. American Journal of Hypertension 2008; 21:521-526.

14 Bhattacharya S, Campbell DM, Smith NC. Pre-eclampsia in the second pregnancy: does previous outcome matter? European Journal of Obstetrics, Gynecology, and Reproductive Biology 2009; 144:130-134.

15 Hernandez-Diaz S, Toh S, Cnattingius S. Risk of pre-eclampsia in first and subsequent pregnancies: prospective cohort study. BMJ 2009; 338:b2255.

16 Luo ZC, An N, Xu HR, Larante A, Audibert F, Fraser WD. The effects and mechanisms of primiparity on the risk of pre-eclampsia: a systematic review. Paediatric and Perinatal Epidemiology 2007; 21 (Suppl. 1):36-45.

17 Miranda ML, Swamy GK, Edwards S, Maxson P, Gelfand A, James S. Disparities in maternal hypertension and pregnancy outcomes: evidence from North Carolina, 1994-2003. Public Health Reports 2010; 125:579-587.

18 Agyemang C, Vrijkotte TG, Droomers M, van der Wal MF, Bonsel GJ, Stronks K. The effect of neighbourhood income and deprivation on pregnancy outcomes in Amsterdam, The Netherlands. Journal of Epidemiology and Community Health 2009; 63:755-760.

19 Clausen T, Oyen N, Henriksen T. Pregnancy complications by overweight and residential area. A prospective study of an urban Norwegian cohort. Acta Obstetricia et Gynecologica Scandinavica 2006; 85:526-533.

20 Gudmundsson S, Bjorgvinsdottir L, Molin J, Gunnarsson G, Marsal K. Socioeconomic status and perinatal outcome according to residence area in the city of Malmo. Acta Obstetricia et Gynecologica Scandinavica 1997; 76:318323. 
21 Auchincloss AH, Diez Roux AV, Dvonch JT, Brown PL, Barr RG, Daviglus ML, et al. Associations between recent exposure to ambient fine particulate matter and blood pressure in the Multi-ethnic Study of Atherosclerosis (MESA). Environmental Health Perspectives 2008; 116:486-491.

$22 \mathrm{Wu}$ J, Ren C, Delfino RJ, Chung J, Wilhelm M, Ritz B. Association between local traffic-generated air pollution and preeclampsia and preterm delivery in the south coast air basin of California. Environmental Health Perspectives 2009; 117:1773-1779.

23 van den Hooven EH, de Kluizenaar Y, Pierik FH, Hofman A, van Ratingen SW, Zandveld PY, et al. Air pollution, blood pressure, and the risk of hypertensive complications during pregnancy: the generation R study. Hypertension 2011; 57:406-412.

24 Miranda ML, Maxson P, Edwards S. Environmental contributions to disparities in pregnancy outcomes. Epidemiologic Reviews 2009; 31:67-83.

25 US Environmental Protection Agency. Air Quality System. 2006. http:/ /www.epa.gov/ttn/airs/airsaqs/ detaildata/downloadaqsdata.htm [last accessed October 2009].

26 Messer LC, Laraia BA, Kaufman JS, Eyster J, Holzman C, Culhane J, et al. The development of a standardized neighborhood deprivation index. Journal of Urban Health 2006; 83:1041-1062.

27 Elo IT, Culhane JF, Kohler IV, O'Campo P, Burke JG, Messer LC, et al. Neighbourhood deprivation and small-for-gestational-age term births in the United States. Paediatric and Perinatal Epidemiology 2009; 23:87-96.

28 Holzman C, Eyster J, Kleyn M, Messer LC, Kaufman JS, Laraia BA, et al. Maternal weathering and risk of preterm delivery. American Journal of Public Health 2009; 99:1864-1871.
29 O'Campo P, Burke JG, Culhane J, Elo IT, Eyster J, Holzman C, et al. Neighborhood deprivation and preterm birth among non-Hispanic Black and White women in eight geographic areas in the United States. American Journal of Epidemiology 2008; 167:155-163.

30 Matheson FI, White HL, Moineddin R, Dunn JR, Glazier RH. Neighbourhood chronic stress and gender inequalities in hypertension among Canadian adults: a multilevel analysis. Journal of Epidemiology and Community Health 2009; 64:705-713.

31 Silva LM, Coolman M, Steegers EA, Jaddoe VW, Moll HA, Hofman A, et al. Low socioeconomic status is a risk factor for preeclampsia: the Generation R Study. Journal of Hypertension 2008; 26:1200-1208.

32 Caughey AB, Stotland NE, Washington AE, Escobar GJ. Maternal ethnicity, paternal ethnicity, and parental ethnic discordance: predictors of preeclampsia. Obstetrics and Gynecology 2005; 106:156-161.

33 Conde-Agudelo A, Althabe F, Belizan JM, Kafury-Goeta AC. Cigarette smoking during pregnancy and risk of preeclampsia: a systematic review. American Journal of Obstetrics and Gynecology 1999; 181:1026-1035.

34 England L, Zhang J. Smoking and risk of preeclampsia: a systematic review. Frontiers in Bioscience 2007; 12:2471-2483.

35 Magnussen EB, Vatten LJ, Lund-Nilsen TI, Salvesen KA, Davey Smith G, Romundstad PR. Prepregnancy cardiovascular risk factors as predictors of pre-eclampsia: population based cohort study. BMJ 2007; 335:978.

36 Gray SC, Edwards SE, Miranda ML. Assessing exposure metrics for PM and birth weight models. Journal of Exposure Science \& Environmental Epidemiology 2010; 20:469-477.

37 Vinikoor LC, Messer LC, Laraia BA, Kaufman JS. Reliability of variables on the North Carolina birth certificate: a comparison with directly queried values from a cohort study. Paediatric and Perinatal Epidemiology 2010; 24:102-112. 